\title{
A Non-Helical Dynamo - MHD Simulations of Dynamo Action by a Non-Helical Flow
}

\author{
V. Archontis \\ Instituto de Astrofisica de Canarias, Via Lactea, E-38200 La Laguna
}

S.B.F. Dorch

The Niels Bohr Institute, Juliane Maries Vej 30, DK-2100 Copenhagen

\begin{abstract}
We illustrate that helicity is not a necessary ingredient for fast dynamo action; we use the stagger-grid method of Galsgaard, Nordlund and others (e.g. Galsgaard \& Nordlund 1997, and applied to dynamos by e.g. Dorch 2000): we solve the full MHD equations including a forcing term that keeps the kinetic energy at an approximately constant level. A 3-d flow with no mean helicity (an ABC-like flow without cosines, cf. Galloway \& Proctor 1992) is implemented and it turns out that apart from the high growth rate in the linear regime (compared to kinematic dynamo action, cf. Archontis \& Dorch 2003a), the dynamo saturates at a level significantly higher that the intermittent turbulent dynamos (cf. Archontis \& Dorch 2003b); namely at exact energy equipartition. During the linear regime, several kinematic modes are present, e.g. a sheet/vortex-mode and a mode that resembles the ABC "double cigar" mode (e.g. Dorch 2000). In the non-linear regime, the magnetic topology is not symmetric, but the initial structure of the velocity field is retained. The presence of helicity is not a requirement for dynamo action but it is rather the stretching ability of the flow that amplifies the magnetic energy in an exponential manner (Archontis \& Dorch, in preparation).
\end{abstract}

A miniature copy (A4) of the poster may be downloaded (MS PowerPoint) from the following URL:

http://www . astro.ku.dk/ dorch/posters/JD03.ppt

Acknowledgments. VA thanks the EU-TMR for support through a Marie Curie Fellowship. SBFD was supported by the Danish Natural Science Research Foundation, through a Steno research grant.

\section{References}

Archontis, V., Dorch, S.B.F. \& Nordlund, A. 2003a, A\&A, 397, 393

Archontis, V., Dorch, S.B.F. \& Nordlund, A. 2003b, A\&A, 410, 759

Dorch, S.B.F. 2000, Physica Scripta, 61, 717

Galloway, D. \& Proctor, M. 1992, Nature, 356, 691

Galsgaard, K. \& Nordlund, A. 1997, J. Geophys. Rev., 102, 219 